\title{
Case Report: Rapidly Growing Intra-Cardiac Mass Mimicking a Local Recurrence of Lung Cancer or a Thrombus in the Left Atrium
}

\author{
Tae Yun Kim, $\mathbf{M D}^{1}$, Kyung Hwa Kim, MD, $\mathrm{PhD}^{1,2}$ \\ ${ }^{1}$ Department of Thoracic and Cardiovascular Surgery, Chonbuk National University Hospital, Jeonju, Korea; ${ }^{2}$ Research Institute \\ of Clinical Medicine of Chonbuk National University-Biomedical Research Institute of Chonbuk National University Hospital, \\ Jeonju, Korea
}

\section{ABSTRACT}

Cardiac myxoma typically is thought to be a slowgrowing, benign primary. Atrial myxomas can lead to many complications and can also mimic mitral stenosis, infective endocarditis, and other vascular diseases associated with systemic embolization. A 75-year-old woman with a history of lung cancer (pT1cN1, adenocarcinoma), atrial fibrillation, and a cerebral infarction presented with dysarthria and visual disturbances. In our case, we had to consider some questionable issues with the left atrial mass, and whether the recurrence of cerebral events was due to the thrombotic material in the left atrium or from locally recurrent lung cancer from the stump margin of the previously resected left superior pulmonary vein. We present a case with a rapidly-growing left atrial myxoma with a growth rate of $12.60 \mathrm{~mm} / \mathrm{month}$, rather than a thrombus or local recurrence of tumor under a medication of nonVKA oral antagonists.

\section{INTRODUCTION}

Cardiac myxoma is the most common type of benign primary cardiac tumor [Reynen 1995]. Typically, cardiac myxomas manifest as a solitary, pedunculated, mobile mass in the left atrium with a stalk that is attached to the interatrial septum. However, due to the relative infrequency of primary cardiac tumors, the diagnosis of cardiac tumors can be challenging, especially if the growth rate is too fast. Also, the clinical presentations characteristics can mimic mitral stenosis, infective endocarditis, and other vascular diseases associated with systemic embolization [Andreua 2013].

Received March 21, 2019; accepted fune 20, 2019.

Author contributor: Tae Yun Kim (e-mail: uadocjon1314@hanmail.net).

Correspondence: Kyung Hwa Kim, MD, PhD, Department of Thoracic and Cardiovascular Surgery, Chonbuk National University Medical School, Chonbuk National University Hospital. Research Institute of Clinical Medicine of Chonbuk National University and Biomedical Research Institute of Chonbuk National University Hospital, 20 Geonji-Ro, Geumam-Dong, Deokjin-Gu, Jeonju, 54907, Republic of Korea; 82-63-250-2522; fax: 8263-250-1480 (e-mail: tcskimgh@gmail.com).
Case Description: A 75-year-old woman was referred to our institution one month ago, due to dysarthria and visual disturbance. She had a medication history of dabigatran to treat atrial fibrillation and a cerebral infarction two years ago. Also, a left upper lobectomy was performed because of lung

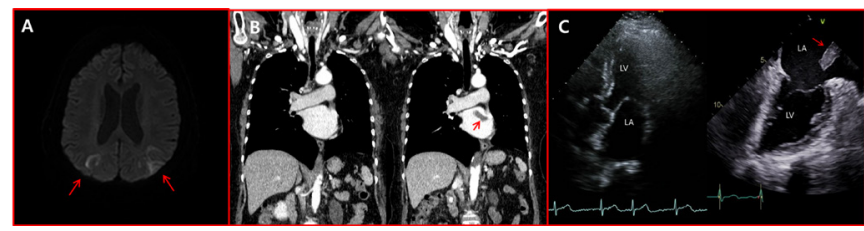

Figure 1. A. Brain magnetic resonance imaging showing the sub-acute infarction in both occipital area (arrow) and multifocal nodular lesion in both white matters. B. Computed tomography, and C. Echocardiography before and three months after a new filling defect appeared in the left atrium (arrow) after a left upper lobectomy.

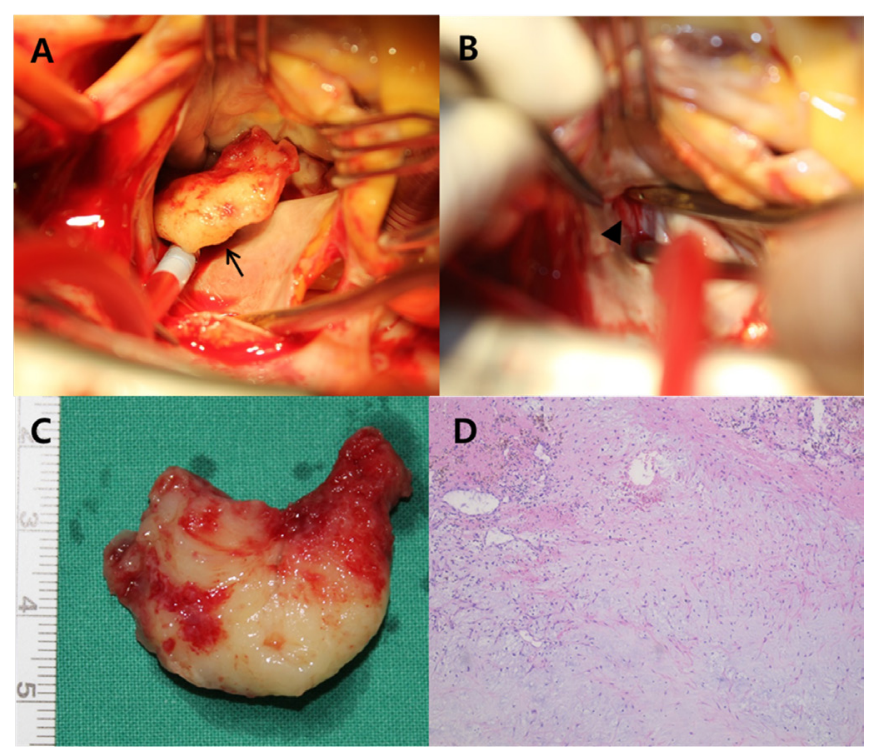

Figure 2. A,B: In the operative finding, a well-organized and free-floating mass was seen in the left atrium (A, arrow), and a clear stump margin ( $B$, arrow head) was left after the mass was removed in a previously resected, left superior pulmonary vein. C,D: Surgical specimen (C) from the resected left atrial mass and pathological findings (D) showed abundant myxoid stroma (hematoxylin \& eosin, original $\times 100$ ). 
cancer (pT1cN1, adenocarcinoma) three months prior. Brain magnetic resonance imaging showed a subacute infarction in both occipital areas (Figure 1A). We evaluated her for distant metastasis and local recurrence of lung cancer. The radiologic investigations revealed a new filling defect in her left atrium (Figure 1B). The 12-lead electrocardiogram (EKG) showed atrial fibrillation, with a rapid ventricular response. A transesophageal echocardiogram demonstrated a highly mobile, homogenous, echogenic and 3.8-cm X 2.7-cm diameter mass originating from the left pulmonary vein, but no thrombus in the left atrial appendage (Figure 1C, Video1). We considered whether or not the recurrent cerebral events were due to the thrombotic material in the left atrium or from locally recurrent lung cancer from the stump margin of the previously resected left superior pulmonary vein. Cardiac surgery was performed one month after the patient's cerebral symptoms improved. We identified a well-organized mass growing from the clear stump margin of the resected left superior pulmonary vein (Figure 2A,B) and performed a Cox-maze procedure with left atrial appendage obliteration. Surprisingly, pathological findings were consistent with a myxoma rather than an organized thrombus and recurrent tumor (Figure 2C,D). The last follow-up postoperative EKG showed normal sinus rhythm, and the patient is doing well without neurologic complication.

\section{DISCUSSION AND CONCLUSIONS}

Primary cardiac tumors are rare with an overall incidence of 0.02 percent [Reynen 1995]. Due to the relative infrequency, the diagnosis of cardiac tumors can be challenging, especially if the growth rate is too fast. Reports with documented growth rate are therefore very rare, and the actual growth rate remains a controversial issue [Jeroen 2010].

In our case, we had to consider some questionable issues with the left atrial mass, and whether the recurrence of cerebral events was due to thrombotic material in the left atrium or from locally recurrent lung cancer from the stump margin of the previously resected left superior pulmonary vein. The presence of atrial fibrillation enlarged atrial chamber, and rapid growth rate of the mass in question were the features characteristic of a thrombus. Also, thrombus formation at the pulmonary vascular resection site is an unusual, but potential complication contributing to recurrent neurological events. Therefore, we thought that our patient's neurological event may have originated from embolization of a cardiac thrombus or a distant metastasis of a local recurrence of the pulmonary tumor. Also, because an organized thrombus associated with an underlying atrial fibrillation may be less likely to respond to lytic or anticoagulation therapy [Hammami 2012; Nobuhiko 2018], we surgically removed the left atrial mass. Surprisingly, in the operative finding, the pathology of the left atrial mass was revealed to be a myxoma, although we thought it was an organized thrombus from the stump margin of the pulmonary vein. In our case, TTE was performed three months prior to cardiac surgery and showed a clear left atrium. Just three months later, a cardiac mass measuring $3.8 \mathrm{~cm}$ in diameter was discovered.

In conclusion, it is worthwhile to point out the discovery of a rapidly-growing atrial myxoma with a growth rate of $12.60 \mathrm{~mm} / \mathrm{month}$ after lung resection rather than a thrombus or local recurrence of tumor under a medication of non-VKA oral antagonists. To the best of our knowledge, our case is the fastest-growing rate in left atrial myxoma in literature.

\section{REFERENCES}

Andreua P, Parrillab G, Arribasa JM, García-Villalba B, Lucas JJ, Garcia Navarro M, Marín F, Gutierrez F, Moreno A. 2013. Neurological manifestations of cardiac myxoma: Experience in a referral hospital. Neurologia. Nov-Dec;28(9):529-34.

Hammami R, Abid L, Mallek S, Kharrat I, Ellouz M, Hentati M, Frikha I, Kammoun S. 2012. A neovascularized left atrial mass. Case Rep Med. 2012:518539.

Jeroen Walpot, Bharati Shivalkar, Inez Rodrigus, W. Hans Pasteuning, Raymond Hokken. 2010. Atrial Myxomas Grow Faster Than We Think. Echocardiography 27:E128-E131.

Nobuhiko Haruki, Takeshi Onohara, Daiki Tsujimoto, Kazuhiko Iitsuka, Yoshiharu Kinugasa, Masahiko Kato, Motonobu Nishimura, Kazuhiro Yamamoto. 2018. Rapid-Growing Right Atrial Myxoma 7 Months After Catheter Ablation Under Anticoagulation Therapy - Serial Echocardiography and Computed Tomography. Circ J. Sep 25;82(10):2682-2683.

Reynen K. 1995. Cardiac myxomas. New Engl J Med. 333(24):1610-7. 